\title{
Can NOx reduction by CO react over carbon-based single-atom catalysts at low temperatures? A theoretical study
}

\author{
Jie Shi ${ }^{1}$, Wei Zhang ${ }^{1}$, Yuan $\mathrm{Pu}^{1}$, Hui Li ${ }^{1}$, Dan Wang ${ }^{1}$, and Jianfeng Chen ${ }^{1}$ \\ ${ }^{1}$ Beijing University of Chemical Technology
}

February 6, 2021

\begin{abstract}
First principles studies combined with the microkinetic analysis were performed to study the reliability and reaction mechanisms of single-atom doped graphene (SADGr) materials in catalyzing NOx reduction with CO. By screening the 3d transition metals ( $\mathrm{Sc}-\mathrm{Zn})$ and group-IV elements ( $\mathrm{Si}$ and $\mathrm{Ge}$ ), it was found that the $\mathrm{Ti}$ and Co doped graphene sheets (TiGr and $\mathrm{CoGr}$ ) respectively own excellent catalytic activities in the NO/NO2-to-N2O and the N2O-to-N2 processes at low temperatures. Therefore, the TiGr/CoGr composite can be a promising catalyst in NOx reduction with CO. It was further revealed the combination of adsorption energy and electronegativity was a good descriptor to predict the activation energies. The obtained results can provide useful information for rational design of carbon-based single-atom catalysts for NOx reduction by $\mathrm{CO}$ at low temperatures.
\end{abstract}

\section{Hosted file}

Manuscript.pdf available at https://authorea.com/users/394162/articles/507613-can-noxreduction-by-co-react-over-carbon-based-single-atom-catalysts-at-low-temperatures-atheoretical-study 
(a)

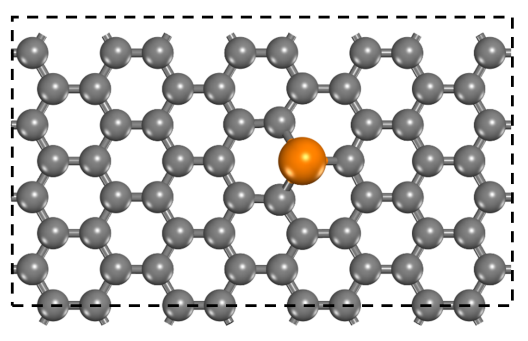

(c)

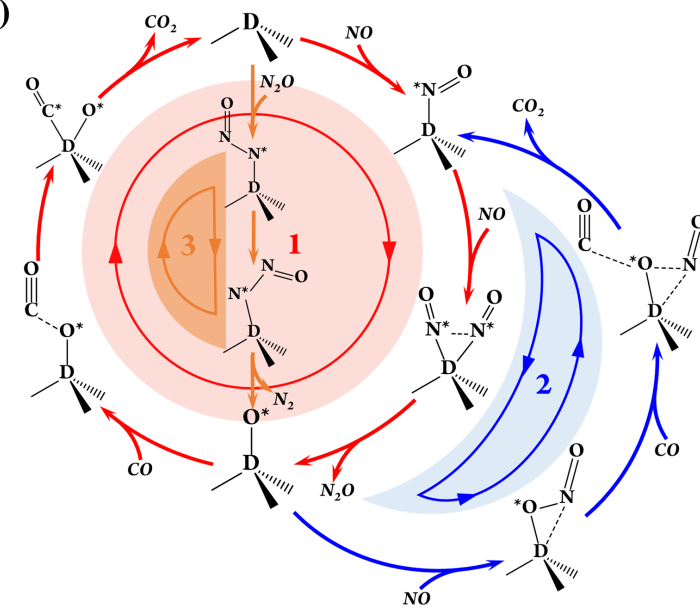

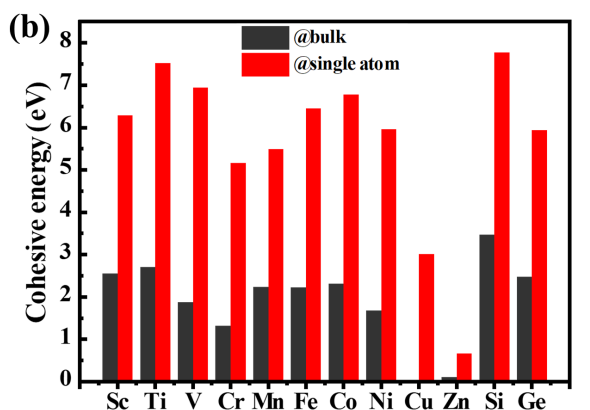

i. $\mathrm{NO}+* \rightarrow \mathrm{NO}^{*}$

ii. $\mathrm{NO}^{*}+\mathrm{NO} \rightarrow(\mathrm{NO})_{2}^{*}$

iii. (NO) $)_{2}^{*} \rightarrow \mathrm{N}_{2} \mathrm{O}+\mathrm{O}^{*}$

iv. $\mathrm{O}^{*}+\mathrm{NO} \rightarrow \mathrm{NO}_{2}^{*}$

v. $\mathrm{NO}_{2}^{*}+\mathrm{CO} \rightarrow \mathrm{NO}^{*}+\mathrm{CO}_{2}$

v(i). $\mathrm{NO}_{2}^{*} \rightarrow(\mathrm{NO}+\mathbf{0})^{*}$

v(ii). $(\mathrm{NO}+\mathrm{O})^{*}+\mathrm{CO} \rightarrow \mathrm{NO}^{*}+\mathrm{CO}_{2}$

vi. $\mathrm{N}_{2} \mathbf{O}+* \rightarrow \mathrm{N}_{2} \mathbf{O}^{*}$

vii. $\mathrm{N}_{2} \mathbf{O}^{*} \rightarrow \mathrm{N}_{2}+\mathbf{O}^{*}$

viii. $\mathrm{CO}+\mathrm{O}^{*} \rightarrow \mathrm{CO}_{2}+*$

viii(i). $\mathrm{CO}+\mathrm{O}^{*} \rightarrow(\mathrm{CO}+\mathrm{O})^{*}$

viii(ii). $(\mathrm{CO}+\mathrm{O})^{*} \rightarrow \mathrm{CO}_{2}+*$
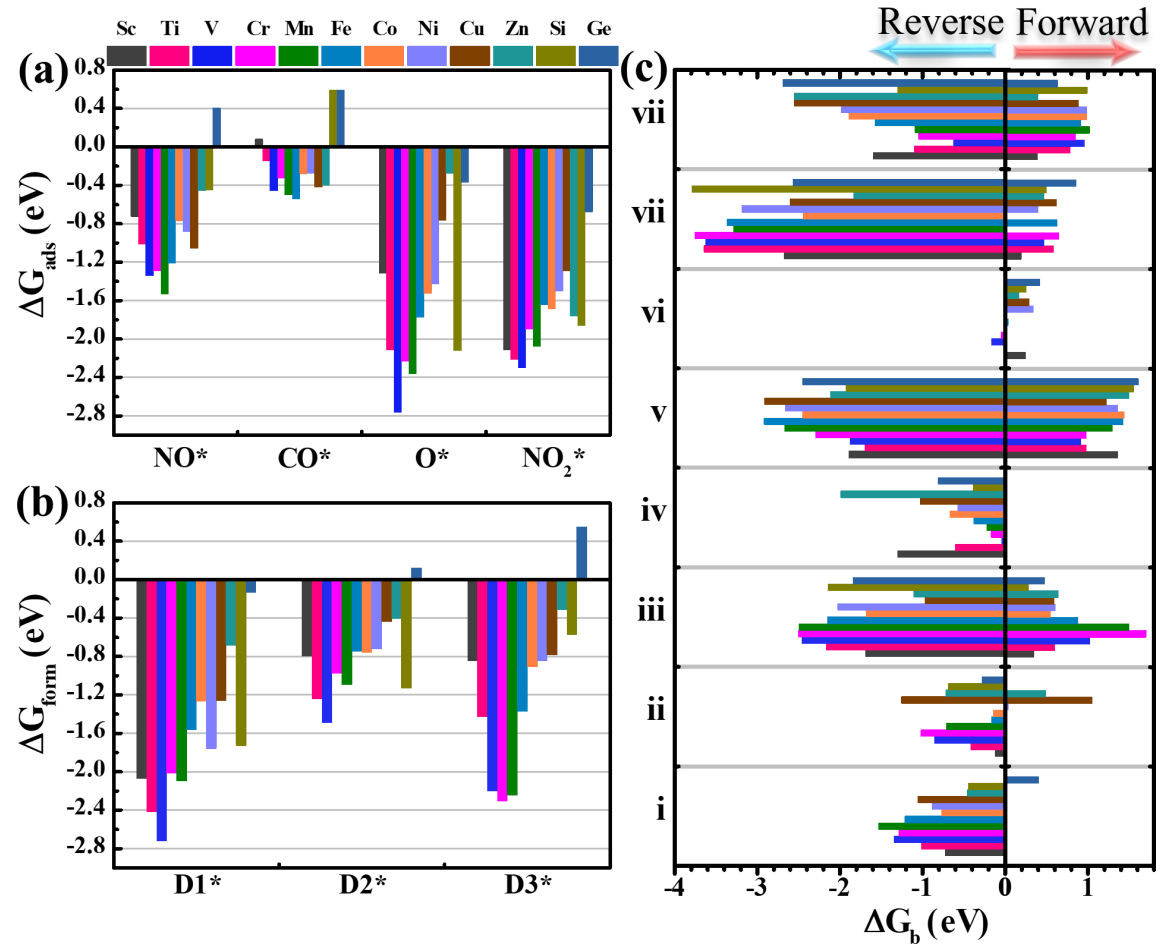

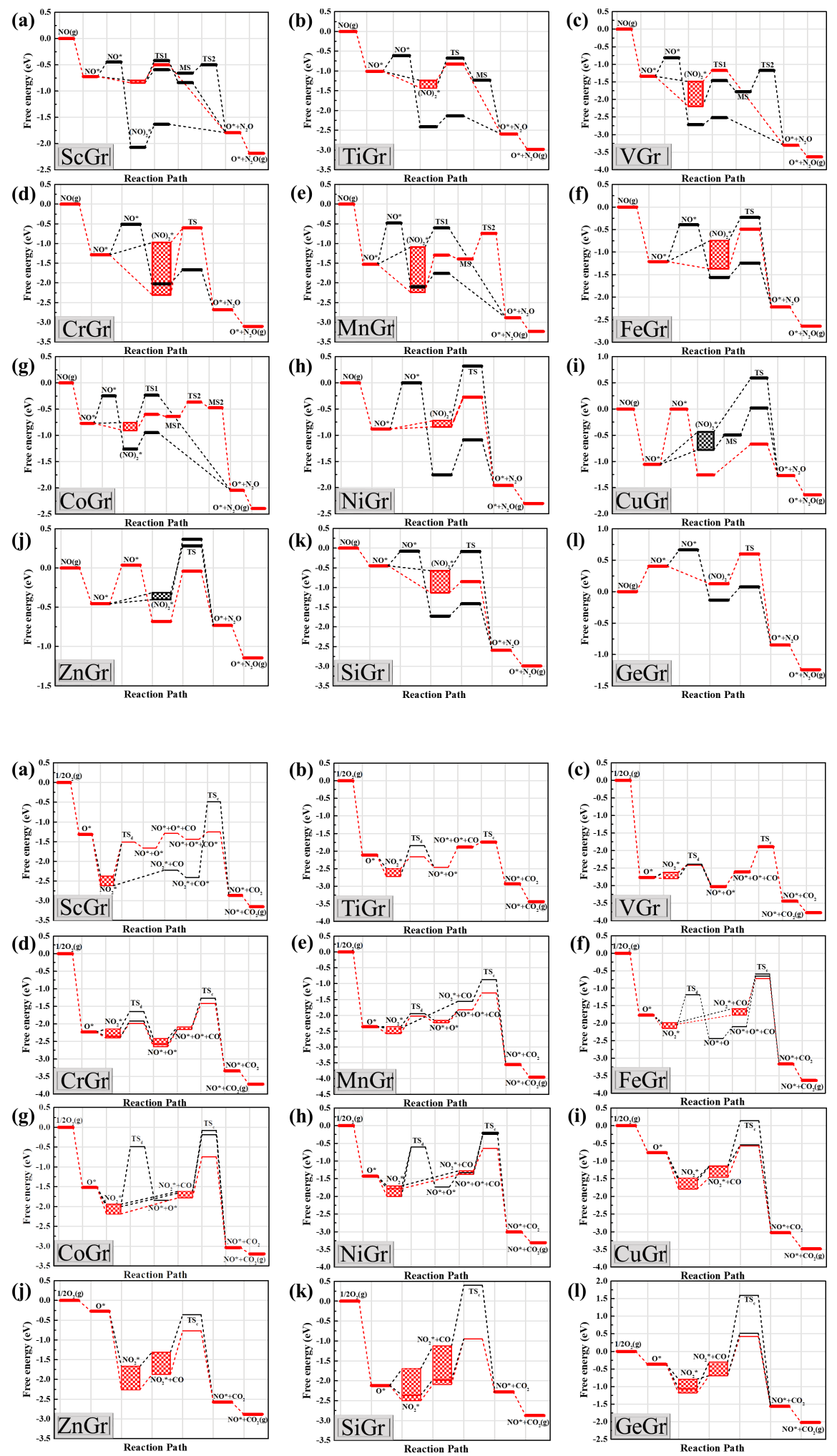

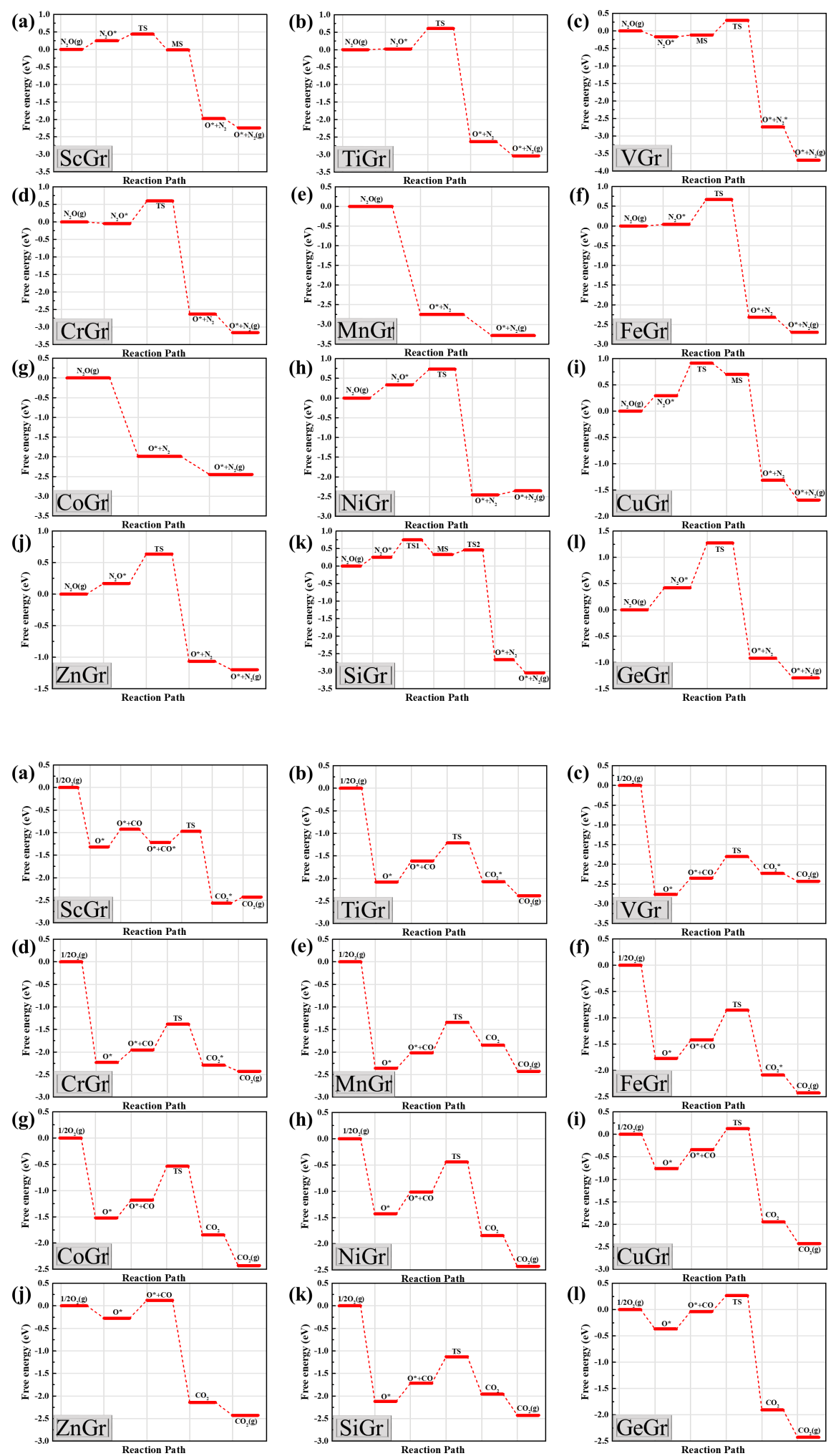


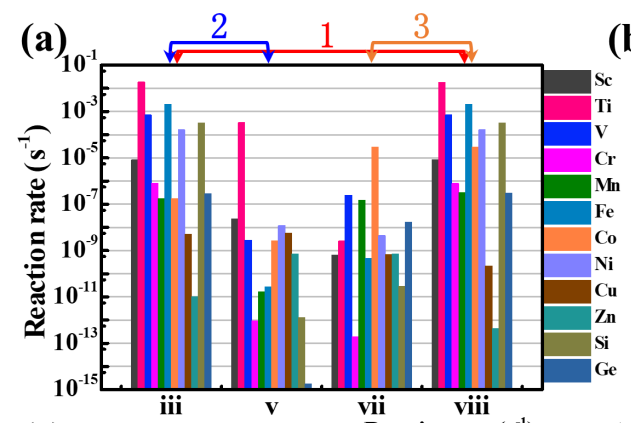

(b)
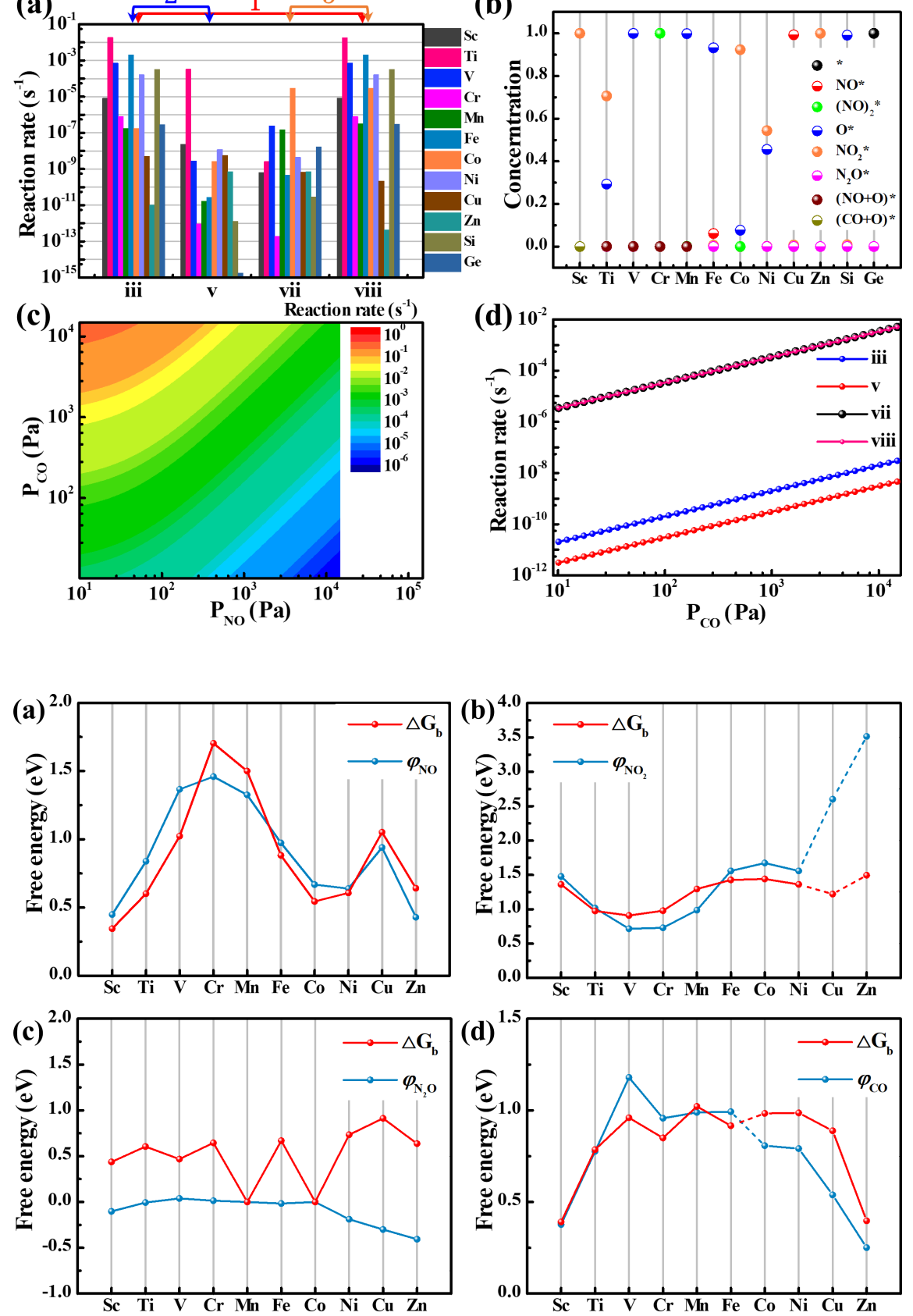

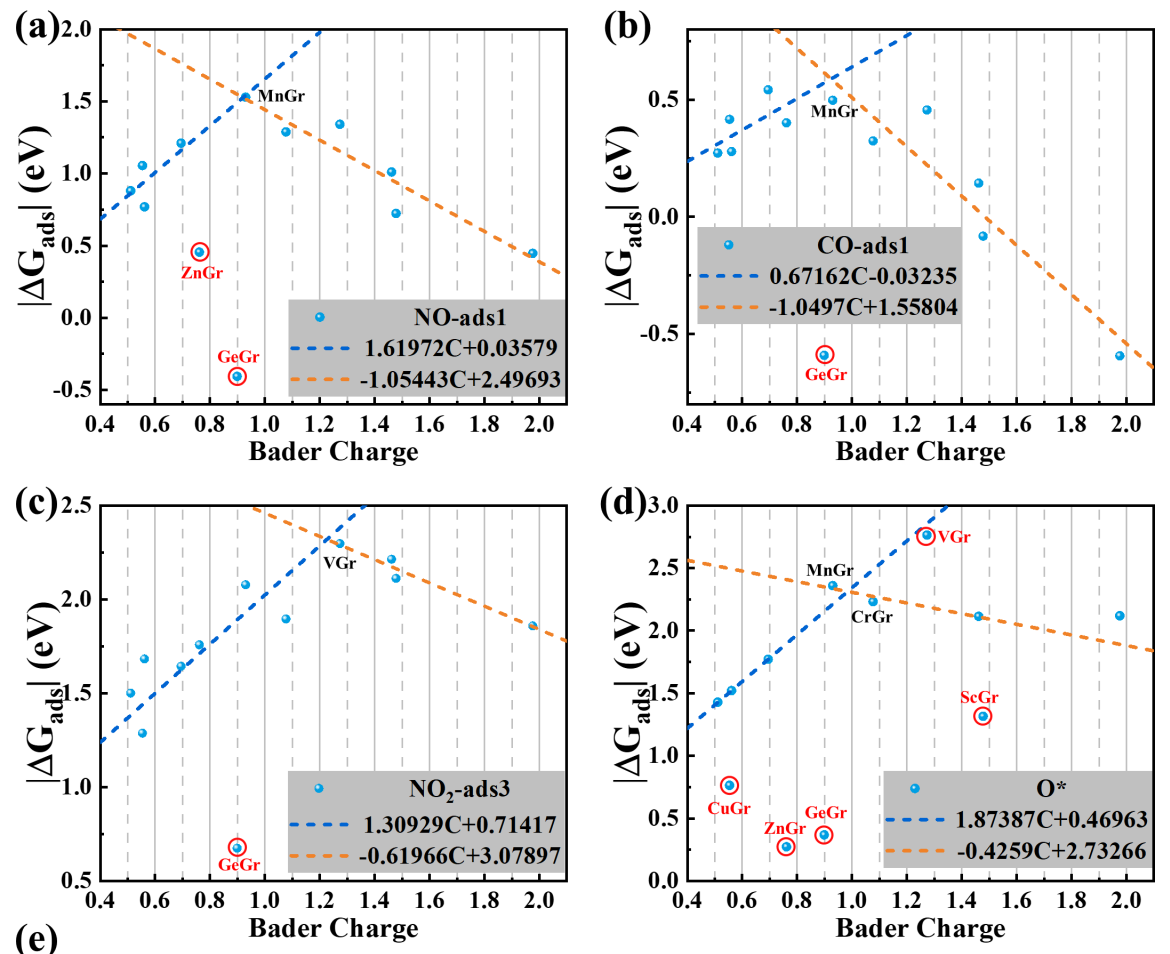

(e)

SADGr NiGr CuGr CoGr FeGr ZnGr GeGr MnGr CrGr VGr TiGr SeGr SiGr

\begin{tabular}{l|llllllllllll} 
Charge & 0.511 & 0.554 & 0.561 & 0.695 & 0.761 & 0.899 & 0.93 & 1.077 & 1.273 & 1.462 & 1.478 & 1.976
\end{tabular} 